\title{
Impacto de la biblioteca universitaria en los estudios de posgrado: análisis de citas de las tesis en tres programas de maestría y doctorado en la Universidad Autónoma de México (UNAM)
}

Impact of academic libraries in postgraduate studies: citation analysis of the PhD thesis of three master and doctoral programmes of the Universidad Autonoma de México (UNAM)

\section{Lucía B. BRito Ocampo (1), María del Pilar LadRón de Guevara Solís (2), Sandra Guadalupe ROSAS POBLANO (3)}

(1) UNAM. Instituto de Investigaciones Biomédicas. Circuito Exterior s/n, CU, México D.F. 04510, lucia@biomedicas.unam.mx (2) UNAM. Facultad de Ciencias. Circuito Exterior s/n, CU, México D.F. 04510 mplgs@hp.fciencias.unam.mx (3) UNAM. Instituto de Química. Circuito Exterior s/n, CU, México D.F. 04510 sandrarp@unam.mx

\section{Resumen}

Se analizaron 5683 citas de 35 tesis de doctorado del año 2009 de los programas de Ciencias Biológicas, Ciencias Biomédicas y Ciencias Químicas. Se identificaron por tipo de documento, edad de la publicación, área temática e idioma. Se determinó la disponibilidad de las fuentes citadas en el Sistema Bibliotecario de la Universidad Autónoma de México (UNAM) por medio de la consulta de los catálogos en línea SERIUNAM y de Revistas Electrónicas contenidos en la Biblioteca Digital. Los artículos científicos fueron los documentos más citados, predominando en gran porcentaje el idioma ingles. La edad promedio de las citas oscila en el quinquenio 2000 a 2004. Se identificaron los 26 títulos más citados en los tres programas. Tanto las bibliotecas sedes de los programas de posgrado como las del Sistema Bibliotecario de la UNAM cuentan con las fuentes de información para apoyar a los estudiantes en el desarrollo de su investigación.

Palabras clave: Análisis de citas. Tesis. Bibliotecas universitarias. Evaluación. Universidad Autónoma de México (UNAM).

\section{Introducción.}

La oferta académica de la Universidad Nacional Autónoma de México (UNAM) en cuanto a los estudios de posgrado es muy amplia, basta mencionar que cuenta con cuatro áreas: ciencias físico-matemáticas y de las ingenierías; ciencias biológicas; ciencias sociales; y humanidades y artes. Dentro de estas áreas existen cuarenta programas de maestría y doctorado (UNAM. Coordinación de Estudios de Posgrado, 2010).

\begin{abstract}
5683 citations from 35 dissertation of the year 2009 of the life Science program, biomedical Science and Chemistry were analyzed. They were identified by document type, date of publication, language and subject area. The availability of the sources cited in the Library System of the Universidad Autónoma de México (UNAM) was checked in the online catalog (SERIUNAM) and Electronic journals contained in the digital library. The most cited articles were mainly of a scientific nature and in English language. The average publishing date of the cited articles was in the range of 2000-2004. The 26 most cited titles in the three programs were identified. It was concluded that both the libraries of the post-graduate centres and those of the UNAM Library System have the necessary sources of information to support students in developing their research.
\end{abstract}

Keywords: Citation analysis. Ph D thesis. Master Thesis. Academic libraries. Assessment. Universidad Autónoma de México (UNAM).

Este trabajo se centra en el área de ciencias biológicas, químicas y de la salud, particularmente en tres programas: Doctorado en Ciencias Biológicas (CB), Ciencias Biomédicas $(\mathrm{CBm})$ y Ciencias Químicas (CQ).

La biblioteca universitaria es el "centro de cualquier proyecto educativo de formación de profesionistas en una universidad" (Lau, 2002). "Es un centro de recursos de información que basa su gestión en la filosofía de la globalización, en el uso de las nuevas tecnologías de información 
y comunicación, en la cooperación y en la calidad" (Orera, 2007).

En la llamada sociedad de la información la biblioteca universitaria se enfrenta a nuevos retos como el valor al acceso a la información. Está obligada a jugar un papel más dinámico y lograr una mayor integración con la misión y objetivos de la universidad coadyuvando a sus funciones sustantivas: docencia, investigación y extensión de la cultura.

\section{Objetivo.}

El presente trabajo tiene como objetivo identificar las fuentes de información que citan los estudiantes de doctorado en sus tesis y determinar la contribución de las bibliotecas por medio de la disponibilidad impresa y electrónica de las fuentes, en las bibliotecas de las sedes de los posgrados y en el sistema bibliotecario de la UNAM.

\section{Metodología.}

Se realizó un análisis de citas de las tesis de doctorado presentadas en tres programas de la UNAM: Ciencias Biológicas, Ciencias Biomédicas y Ciencias Químicas en el año 2009. Se eligió una muestra de las tesis recibidas en las bibliotecas sedes de los posgrados: Facultad de Ciencias, Instituto de Investigaciones Biomédicas e Instituto de Química de la UNAM.

En total se revisaron 35 tesis obteniendo 5683 referencias, las cuales se analizaron por tipo de documento, revista, edad de la publicación, categoría por área temática e idioma. Se determinó la disponibilidad de las fuentes en el Sistema Bibliotecario de la UNAM en formato impreso y/o electrónico.

Se utilizaron Microsoft Access y Excel para el manejo de la información. Se identificaron las fuentes de información y se clasificaron en las siguientes categorías: artículos científicos, monografías, series y otros, agrupando en esta última las tesis, recursos electrónicos, actas de conferencias, patentes, reportes y demás materiales citados.

Para categorizar las disciplinas a las que pertenecen las revistas se consultó el Journal Citation Reports (ISI, 2008). Con el fin de saber si las revistas se encuentran en las bibliotecas de las sedes de los programas de posgrado o en el Sistema Bibliotecario de la UNAM se consultaron los catálogos en línea SERIUNAM y de Revistas Electrónicas contenidas en la Biblioteca Digital.

\section{Revisión de la literatura.}

De acuerdo con Fernández, (2006) "una tesis doctoral debe realizar una completa y ordenada revisión de la literatura tanto conceptual como de investigación que exponga los hallazgos más recientes".

Los análisis de citas a revistas se remontan al año 1927 (Gross y Gross, 2009) en revistas del área química, sin embargo los trabajos de estudiantes se analizaron a partir de los 90's. Por su parte Zipp (cit. por ibidem), encontró una correlación entre las citas de los estudiantes y los profesores e investigadores, incluso los hallazgos pueden ser extrapolados a otro grupo de usuarios.

Artículos de esta naturaleza están reportados en la literatura (livonen, 2009) al estudiar la comparación de la disponibilidad de referencias en 10 tesis de doctorado en dos universidades de Finlandia en las áreas de negocios y administración en el periodo 2005-2006. Localizó 3194 citas de las cuales el $82 \%$ de los artículos estuvieron disponibles en la biblioteca de una Universidad y $92 \%$ en otra, en cuanto a los accesos electrónicos reportó el $68 \%$ en una y el $79 \%$ en otra universidad.

Vallmitjana (2008) realizó un estudio bibliométrico de las referencias en 26 tesis de doctorado en el área de química en el periodo 1995-2003. Analizó 4203 , de ellas el $79 \%$ fueron artículos, 33 revistas representaron el $50 \%$ de las necesidades de información, el $50 \%$ de los artículos tuvo una edad no mayor de nueve años.

En otro estudio similar, pero en el área de educación matemática en universidades españolas (Fernández, 2006) se revisaron las referencias citadas en 241 tesis doctorales en el periodo 1975-2002. El autor obtuvo en promedio 200 referencias por tesis. El idioma inglés fue el idioma más utilizado y el segundo el español. Encontró que los libros fueron las fuentes más citadas, lo que no ocurre con las áreas de ciencias puras de acuerdo a la literatura revisada. En cuanto a la antigüedad la edad promedio de las referencias fue de 12 años. Destaca haber encontrado errores y distorsiones en la presentación de las citas.

Sinn (2005) estudió 67 tesis doctorales presentadas en la Universidad de Ohio en el periodo 1980 a 2002. Encontró 1413 artículos publicados en 299 títulos de revistas. Sus resultados muestran que los artículos representan el $65.9 \%$, con un promedio de edad de 16.6 años y el de libros fue de 14.5. Analizó los patrones de cita entre disciplinas en donde la química y la biología utilizan más artículos científicos, en 
comparación con las matemáticas, estadística e ingeniería, en donde utilizan más monografías.

\section{Resultados}

De las 35 tesis analizadas se obtuvieron 5683 citas, lo cual da en promedio 140 referencias por tesis.

En la tabla I se muestran 5 categorías del ISI con mayor representatividad. Se destaca la categoría de ciencias multidisciplinarias en los tres programas. En el área de ciencias biomédicas destaca la categoría de bioquímica y biología molecular e inmunología; en el área de ciencias biológicas biotecnología y microbiología aplicada, y ecología. En el área de ciencias químicas sobresalen las categorías química inorgánica y química orgánica.

\begin{tabular}{|c|c|c|}
\hline \multicolumn{3}{|c|}{ Áreas del ISI } \\
\hline $\begin{array}{l}\text { Ciencias } \\
\text { biomédicas }\end{array}$ & $\begin{array}{l}\text { Ciencias } \\
\text { biológicas }\end{array}$ & $\begin{array}{l}\text { Ciencias } \\
\text { químicas }\end{array}$ \\
\hline $\begin{array}{l}\text { Ciencias } \\
\text { Multidisciplinar } \\
\text { ias } \\
\end{array}$ & $\begin{array}{l}\text { Biotecnología y } \\
\text { Microbiología } \\
\text { Aplicada }\end{array}$ & $\begin{array}{l}\text { Química } \\
\text { Multidisciplinaria }\end{array}$ \\
\hline $\begin{array}{l}\text { Bioquímica y } \\
\text { Biología } \\
\text { Molecular }\end{array}$ & $\begin{array}{l}\text { Ciencias } \\
\text { Biológicas }\end{array}$ & $\begin{array}{l}\text { Química } \\
\text { Inorgánica }\end{array}$ \\
\hline Inmunología & $\begin{array}{l}\text { Ciencias } \\
\text { Multidisciplinaria } \\
\mathrm{s}\end{array}$ & $\begin{array}{l}\text { Química } \\
\text { Orgánica }\end{array}$ \\
\hline Neurociencias & Ecología & Química Física \\
\hline $\begin{array}{l}\text { Biología } \\
\text { Celular }\end{array}$ & $\begin{array}{l}\text { Ciencias } \\
\text { Ambientales }\end{array}$ & $\begin{array}{l}\text { Ciencias de Las } \\
\text { Plantas }\end{array}$ \\
\hline
\end{tabular}

Tabla I. Categoría por áreas con mayor número de citas en cada programa.

Los documentos más citados fueron los artículos científicos, seguidos de las monografías, y en menor porcentaje las tesis, patentes, actas de conferencias, reportes y recursos electrónicos (Fig. 1). El idioma más citado fue el inglés lo cual pudo comprobarse con otros estudios citados con anterioridad, en segundo término el español, coincidiendo en resultados similares realizados en universidades españolas (Fig 2).

La edad promedio de las citas coincidió en el intervalo de los artículos publicados en el periodo 2000 a 2004, es decir en su mayoría los artículos más citados tienen una edad de cinco a nueve años.

Los años más citados en segundo término fueron los artículos publicados del 2004 a la fecha y en un porcentaje reducido los artículos de las décadas de los setentas hacia atrás, esto últi- mo, posiblemente por tratarse de citas clásicas a la literatura de la especialidad (Fig. 3).

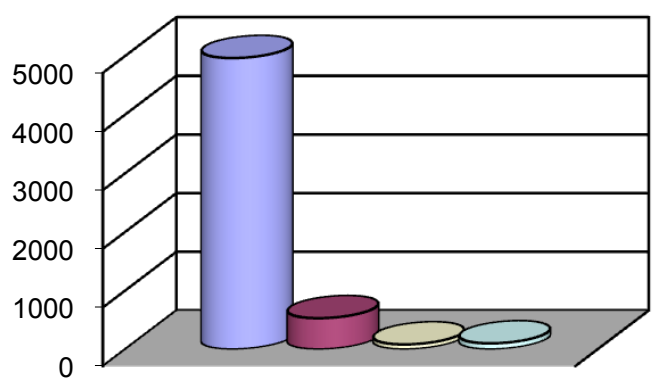

Fig. 1. Distribución por tipo de documento.

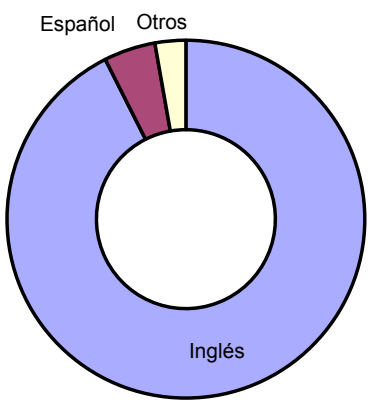

Fig. 2. Idioma de las referencias.

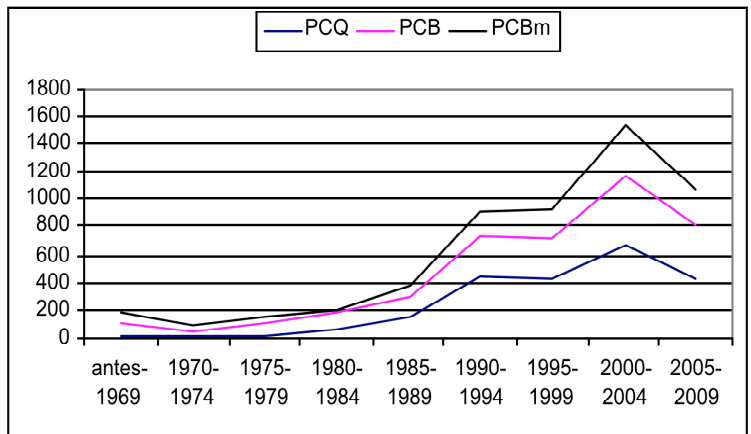

Fig. 3. Distribución por edad de las referencias

Las revistas de carácter multidisciplinario como Science y Nature fueron de las veinte revistas más citadas en los los tres programas. El título más citado en el Programa de Ciencias Biológicas fue Mutation Research, en el Programa de Ciencias Biomédicas Journal of Biological Chemistry y en el Programa de Ciencias Químicas el título más citado fue Journal of the American Chemical Society.

Se identificaron 1172 títulos de revistas científicas, de éstas el $87.88 \%$ se encontró en formato impreso y/o electrónico en el Sistema Biblioteca- 
rio de la UNAM. En cuanto a libros el $61.43 \%$ se localizó en las bibliotecas del sistema (Fig. 4).

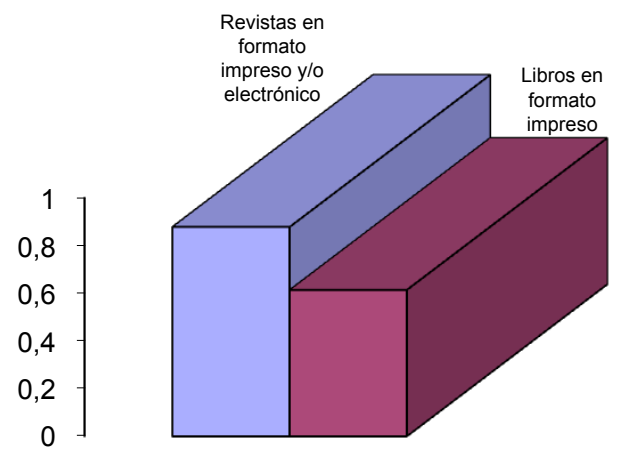

Fig 4. Porcentaje de disponibilidad en el Sistema Bibliotecario.

\begin{tabular}{lr}
\hline Titulo de la Revista & Citas \\
\hline Mutation Research & 126 \\
\hline Journal of the American Chemical Society & 118 \\
\hline Nature & 104 \\
\hline Proceedings of the National Academy of Sciences & 102 \\
\hline Science & 100 \\
\hline Journal of Biological Chemistry & 97 \\
\hline Journal of Virology & 69 \\
\hline Journal of Immunology & 60 \\
\hline Angewandte Chemie International Edition & 54 \\
\hline Inorganic Chemistry & 51 \\
\hline Journal of Bacteriology & 51 \\
\hline Infection Immunology & 41 \\
\hline Journal of Neuroscience & 38 \\
\hline Organometallics & 37 \\
\hline Cell & 36 \\
\hline Journal of Organic Chemistry & 36 \\
\hline Phytochemistry & 34 \\
\hline Chemical Communications & 29 \\
\hline Chemical Reviews & 25 \\
\hline Dalton Transactions & 25 \\
\hline Evolution & 24 \\
\hline Icarus & 22 \\
\hline Journal Geophysical Research Planets & 20 \\
\hline Journal of Infectious Diseases & 20 \\
\hline Tetrahedron & 20 \\
\hline Copeia & 19 \\
\hline
\end{tabular}

Tabla II. Revistas más citadas en los Programas CB, $C B m$ y $C Q$ en orden alfabético.

En la tabla II se muestran los 26 títulos más citados en los programas, cuya disponibilidad fue del $100 \%$ en papel y el $93 \%$ en formato electrónico.

\section{Conclusiones}

Este tipo de análisis ayuda en la toma de decisiones para el desarrollo de las colecciones, ya que se obtienen indicadores para la adquisición o renovación de la suscripción de títulos más citados y en la cancelación de aquellos pobremente consultados.

La biblioteca universitaria cumplirá con su misión en la medida que satisfaga las necesidades académicas de su institución lo cual, se puede medir por la calidad de sus colecciones, en la organización de sus procesos y en el ofrecimiento de servicios oportunos a sus usuarios.

Se detectaron algunos errores y omisiones en las referencias, no obstante, en general los estudiantes de posgrado observan las normas para la redacción de las citas bibliográficas. En la revisión de las mismas se pudo comprobar que los programas como Endnote y Reference Manager son muy utilizados en la preparación de las bibliografías.

Tanto las bibliotecas sedes de los Programas de Posgrado como las del Sistema Bibliotecario de la UNAM cuentan con las fuentes de información para apoyar a los estudiantes en el desarrollo de su investigación.

\section{Referencias}

Fernández, Antonio; Torralbo, Manuel; Vallejo, Mónica (2006). Patrones de citación en la investigación española en educación matemática. // Revista Española de Documentación Científica. 29:3 (2006) 382-397.

Gross y Gross, cit. por Sherriff, Graham (2009). Information use in history research: a citation analysis of Master's level theses at Southern Connecticut State University. Thesis Master of Library Science.

livonen, Mirja; et al. (2009). Library collections contribute to doctoral studies. // Library Management. 30:3 (2009) 185-203.

Journal Citation Reports Science Ed. USA: Thomson Reuters, 2010. http://isiknowledge.com/?DestApp=JCR (2010-02-13)

Lau, Jesús (2002). Bibliotecas universitarias: su importancia en el proceso de acreditación. // Gaceta Universitaria. 17:149 (2002) 8

Orera-Orera, Luisa (2007). La biblioteca universitaria ante el nuevo modelo social y educativo. // El Profesional de la Información. 16:4 (2007) 329-337.

SERIUNAM: revistas de las bibliotecas de la UNAM y de instituciones de educación superior e investigación. México: UNAM, Dirección General de Bibliotecas, 2010. http://132.248.9.4:8991//F/-/?func=find-b-0\&local_base= SER01 (2010-02-14)

Sinn, Robin (2005). A local citation analysis of mathematical and statistical dissertations. // Science and Technologies Libraries. 25:4 (Mayo 2005) 25-37.

UNAM. Coordinación de Estudios de Posgrado. Oferta educativa: programas de posgrado, 2010. http://www. posgrado.unam.mx/programas/index.php (2010-06-14) 
Vallmitjana, Nuria; Sabate, Lucino (2008). Citation analysis of Ph.D. dissertation references as a tool for collection management in an academic chemistry library. // College \& Research Libraries. 69:1 (2008) 72-81.

Zipp, cit. por Sheriff, Graham, idem.

Recibido: 21-05-2010. Aceptado: 21-05-2010.

Brito Ocampo, Lucía B.; Ladrón de Guevara Solís, María del Pilar; Rosas Poblano, Sandra Guadalupe. Impacto de la bibioteca universitaria en los estudios de posgrado. // Ibersid. (2010) 195-199. ISSN 1888-0967. 
